\title{
The impact of GSM-R on railway capacity
}

\author{
D. N. Jansen ${ }^{1}$, S. G. Klabes ${ }^{2}$ \& E. Wendler ${ }^{2}$ \\ ${ }^{1}$ Institute for Computing and Information Sciences, \\ Radboud University, Nijmegen, The Netherlands \\ ${ }^{2}$ Institute of Transport Science, RWTH Aachen University, Aachen, \\ Germany
}

\begin{abstract}
The operation of railway systems strongly depends on the underlying train control system. ERTMS (European Rail Traffic Management System) is a project launched by the European Union in order to increase the interoperability of the national railway systems in Europe. One of the two main components of ERTMS is GSM$\mathrm{R}$, a wireless communication standard based on GSM.

In this paper, we focus on the stochastic nature of GSM-R communication failures and their possible impact on railway capacity. Firstly, we will compare the results of our newly introduced model to the results obtained with a standard blocking time model, applied by a couple of European railway infrastructure managers. After this validation of our model, we will then use the stochastic approach in order to evaluate the impact of GSM-R communication on the railway operation and railway capacity with ETCS level 3 . We can show that ETCS level 3 indeed leads to a capacity increase in our setting. So, while a single GSM-R message may be more error-prone than traditional communication, the framework of ETCS can cope well with this imperfection.
\end{abstract}

Keywords: ERTMS, GSM-R, ETCS level 3, railway capacity.

\section{Introduction}

Railway systems know a long history of train protection and control, as to reduce the risk of train accidents. Many systems include some automated communication between train and trackside equipment. Several different, mostly national systems 
emerged [1]. The different train control systems are still a major obstacle for crossborder rail traffic. Today, trains for cross-border traffic need to be equipped with all train control systems installed on the tracks that the train utilises during its journey. The European Rail Transport Management System (ERTMS) shall lead to a harmonisation of the European train control systems. It is one of the backbone projects to achieve higher interoperability between the different train control systems used in European countries in the hope to increase the share of rail transport on the overall transport in Europe.

ERTMS consists of two standards: the European Train Control System ETCS and the Global System for Mobile communication for Railway applications GSM-R [2]. Today ETCS is foreseen to have three levels (1, 2 and 3). Level 1 defines a standard for discontinuous train control with standardised hardware. Level 2 replaces traditional line side signals by transmission of movement authorities via GSM-R communication. It still operates on an infrastructure that is segmented into fixed blocks. Wendler [3] and Geiß [4] show that adapting the size of these fixed blocks increases the capacity of a line. ETCS level 3 further requires that trains report to the train control centre via GSM-R which infrastructure they have safely left. This provides the possibility to create a virtual block around a train. If GSM-R communication fails, the train control centre cannot reassign the infrastructure to another train and line capacity decreases.

As with many new developments, it is still difficult to estimate the impact of ERTMS. Will it achieve at least the same level of safety as traditional train control? Will it allow at least the same performance (speed, headway)?

\subsection{Formal methods for early evaluation}

Formal modelling, simulation and verification is a method to evaluate the safety and performance of (a model of) a system before its full deployment. Already in an early stage of the development process, one may be able to generate a formal model of the system to be constructed, which then can be fed into several simulation tools. Formal modelling is an accepted method for the evaluation of communication protocols. A formal model also captures unforeseen interference between the parts and exposes weaknesses of the protocol.

However, the results from simulation only carry over to the real system if the modelling process did not introduce some distortion into the model. One of the authors has previously worked on simulation of the GSM-R communication between train and (trackside) block centre in ETCS. To test whether the model is faithful, though, requires expertise from railway engineering. Therefore, we undertook to compare a model based on Jansen and Hermanns [5] (called "Model B") with an equivalent model ("Model A") constructed in the approved railway modelling tool RUT [6]. Model A is used by a couple of European railway managers. This comparison has given us confidence that the earlier model is realistic enough. With a correct model of the communication, one is able to estimate the impact of communication failures on the capacity. 


\subsection{Examining the impact of GSM-R communication}

In order to examine the impact of GSM-R on railway operation, it is necessary to model the communication together with the relevant environment. Our current model thus covers the radio communication, the train behaviour and the track properties.

Wireless GSM-R communication is relatively unreliable, so transmission errors and short blackouts are current. Cell hand-overs (switching from one GSM radio cell to another) are another source of short connection interruptions. Cell sizes are also distributed probabilistically. A realistic model of ERTMS, therefore, has to include a probabilistic model of the radio communication.

The ERTMS specification contains requirements on the minimum quality that GSM-R has to offer [7,8], mostly through bounds on the probability of failure or delay. We assumed that the GSM-R communication quality reaches the prescribed level. (This can be achieved by installing enough base transceiver stations along the track that have enough transmission power.)

In our earlier work [5,9], we checked whether these requirements on the quality of a single transmission ensure an acceptable long-term behaviour. We could show that the small probabilities of failure of single transmissions do not sum up too much during a typical train trip.

The relevant environment of GSM-R, for our purposes, consists of train behaviour and track properties. From the train behaviour, the main elements are the acceleration and braking characteristics. We have chosen characteristics for a high-speed train that reacts quickly to commands of the driver. The track also influences (positive and negative) accelerations that can be achieved; therefore, a choice of track model is necessary.

Unfortunately, the current railway modelling tools like RUT do not allow for probabilistic modelling of communication failures. Due to the different modelling paradigms merging the deterministic Model A and the stochastic communication model is impossible. Therefore the existing probabilistic model of communication [5] was extended by components that simulate the train behaviour and track properties, similarly to Model A. This enabled us to compare the results of both models and to examine the impact of GSM-R communication failures on railway operation.

In particular, the impact of communication failures on the minimum headway time of two similar trains will be discussed.

\section{Simulation approaches}

In this section, we introduce two modelling approaches: a new, stochastic modelling approach and the deterministic model that is used by a couple of European railway infrastructure managers. We examine an example railway system by means of these two approaches and illustrate the obtained results. 


\subsection{Common setup}

In both models, we assumed a single track between two stations $250 \mathrm{~km}$ apart from each other. The track is flat and straight, so the same forces apply to the train everywhere. Start and end stations have two tracks each, connected with a switch. We assumed that two trains run from the start station to the end station in short succession. The trains run at limited speed in the stations and on the switches $(80 \mathrm{~km} / \mathrm{h})$ and are allowed full speed on the track $(250 \mathrm{~km} / \mathrm{h})$. The acceleration and braking characteristics are similar to that of a modern high-speed train. Trains measure their position with an error bound of $50 \mathrm{~m}$. The safe distance between trains is maintained by ETCS level 3 with moving blocks.

\subsection{Deterministic simulation (Model A)}

The deterministic simulations are conducted using Model A. They base on the running time estimation documented by Brünger and Dahlhaus [10] and operate on a microscopic railway model, the so-called Spurplan model. It contains all those components that influence the speed profile of the train and thus its running time. The speed profile is determined by two groups of parameters. One group determines the characteristics of the train (accelerating and braking characteristic). The other group enfolds the track side components and limitations induced by the track: distances, gradients, signal positions, speed limits, etc.

The software tool RUT has been designed with the fixed block operation principle in mind. As described by Wendler [3], the moving block operation principle can be emulated by applying the fixed block operation principle with an infinitesimal length of track segments. Similarly, we simulate moving block operation by positioning a huge number of signals along the track.

Model A supposes a deterministic behaviour of the railway system's components. Trains will run exactly on schedule. This is a useful assumption and deterministic simulations are widely applied in the field of operations research for railway systems. Nevertheless, the assumption of deterministic behaviour is rather strong, since various influences on railway operation can not be modelled this way. One of these influences with uncertain effect is the communication delay when using ETCS level 2 or 3. Therefore, we introduce a stochastic model.

\subsection{Stochastic simulation (Model B)}

We modelled the communication protocol of GSM-R at a high level using the StoCharts modelling language [11], an extension of UML state-charts that includes probabilistic choice and stochastic delays. In particular, we were able to include stochastic communication delays and connection interruptions in accordance with the specification documents. For example, it is prescribed [7] that a message arrives within at most $0.5 \mathrm{sec}$ with probability 0.95 , and within at most $1.2 \mathrm{sec}$ with probability 0.99 . More stochastic requirements can be found in Jansen and Hermanns [5]. We translated the communication model using a prototype translator 
to the MoDeST language [12] and analysed it earlier [5,9]. To make this model more realistic, one has to extend it with a track model. We added the track model mentioned in section 2.1 directly in MoDeST. We chose a very simple track model because the general stochastic language MoDeST does not offer basic operators for track-specific features.

All MoDeST models are analysed by the tool set MoTor / Möbius [13], a discrete event simulator tool that generates statistical overviews from its simulation runs.

\subsection{Comparing the models}

Model A is deterministic. The pre-calculated average communication delay is taken as the communication time. The strength of model B lies with the communication model: the failures are modelled in detail and with realistic random distributions.

The tool RUT (used for model A) allows a detailed track description, with several types of track, points etc. Model B only includes a simple track and train model. To reduce the differences between the models to what we want to compare, we restricted ourselves to an equivalent track and train model in model A, not using all the possibilities of RUT. As mentioned earlier, we can emulate moving block operation by making blocks smaller than the (assumed) measurement error.

As we restricted ourselves to a track and train characteristics that could be described in both models, the only real difference lies in the communication model.

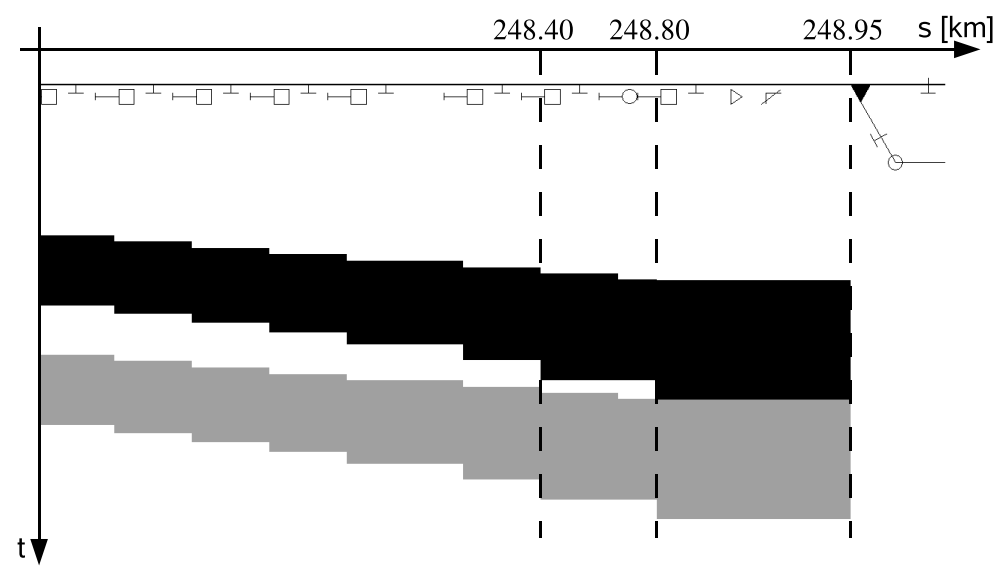

Figure 1: Diagram showing a segment of the track model and the blocking time stairway of two trains following each other with minimum headway time (diagram not to scale). 


\section{Experiment: outcomes and interpretation}

\subsection{Model A: simulation results}

The deterministic simulation conducted with model A calculates the mean running times of both trains on the infrastructure of our example. Moreover, it estimates the blocking times of each train on the sections of the regarded infrastructure under the assumption that the train is not hindered in its run by other trains (green wave). This way the user obtains a blocking time stairway for each train. With this stairway at hand it is possible to evaluate the minimum headway time between the trains by shifting them to the closest possible position without blocking time overlaps. Figure 1 illustrates the blocking time stairways for two trains following each other by a time difference equal to the minimum headway: the stairways touch each other at the last section of the open track. The resulting minimum headway time of the two trains is $110 \mathrm{sec}$ for the deterministic simulation.

\subsection{Model B: single run analysis}

Model B follows a different scheduling principle: the following time, the time between the departures of the two trains, can be chosen freely by the experimenter. Whenever the leading train communicates its new position to the train control system, the freed track is reserved for the second train at once. The train departs after its following time has passed, but if it is too short, the train adapts dynamically by braking somewhere on the track. If the following time is large, the track reserved ahead of the second train is longer than strictly necessary, giving it a buffer against temporary GSM-R failures. Möbius generates a similar blocking time stairway to that in Figure 1 for the two trains including the dynamic adaptation of the following train. We can see that the stairway of the second train is not deformed whenever the following time chosen is at least $111 \mathrm{sec}$.

Using smaller following times, we made an interesting observation: actually, shorter headways upon arrival in the final station are possible. As long as the following time is not much smaller than $111 \mathrm{sec}$, the braking phase is still critical. Before braking starts, the trains can typically achieve a headway around $70 \mathrm{sec}$, and during braking, the headway increases further, but not to $111 \mathrm{sec}$. The reason is that the braking distance (which is the largest part of the distance between the two trains) diminishes quadratically as the following train brakes, but the headway increases less. This effect becomes visible in moving block operation because here, very small (infinitesimal) blocks are possible.

\subsection{Validation of Model B by means of Model A}

In model B, the minimal headway where the following train is unhindered is almost the same as in model A. The blocking time stairways are similar throughout the track. If we start the second train early, it will have to brake on the open track. We could mimic this behaviour in model $\mathrm{A}$, by adding extra speed limitations to the 
Table 1: Positions of the speed limits.

\begin{tabular}{cc}
\hline Position $[\mathrm{km}]$ & Speed $[\mathrm{km} / \mathrm{h}]$ \\
\hline 244.178 & 240 \\
245.771 & 211 \\
247.824 & 136 \\
\hline
\end{tabular}

Table 2: Simulation results.

\begin{tabular}{lcccc}
\hline Following time & $70 \mathrm{sec}$ & $80 \mathrm{sec}$ & $100 \mathrm{sec}$ & $111 \mathrm{sec}$ \\
Mean headway & $78.45 \mathrm{sec}$ & $84.62 \mathrm{sec}$ & $101.28 \mathrm{sec}$ & $111.41 \mathrm{sec}$ \\
$90 \%$ confidence interval & $0.007 \mathrm{sec}$ & $0.003 \mathrm{sec}$ & $0.002 \mathrm{sec}$ & $0.0008 \mathrm{sec}$ \\
Early breaking & $2390 \mathrm{~m}$ & $1162 \mathrm{~m}$ & $447 \mathrm{~m}$ & $0 \mathrm{~m}$ \\
& $28 \mathrm{sec}$ & $21 \mathrm{sec}$ & $10 \mathrm{sec}$ & $0 \mathrm{sec}$ \\
\hline
\end{tabular}

following train that let it brake early, similarly to the early braking phase in model B. We added three extra speed limiting points, as shown in table 1 . The positions and speeds are chosen from a typical simulation run for model A with a small following time $(\leq 80 \mathrm{sec})$ between the trains. By imposing this speed profile on the following train, the minimum headway time is reduced to $92.5 \mathrm{sec}(1.54 \mathrm{~min})$. This minimum headway time does compare to the result obtained by applying Model B. It thus validates Model B.

\subsection{Model B: statistics over runs}

In a second simulation setup, we let MoTor/Möbius collect data from 5.000 simulation runs each for several initial headways to estimate the distribution over the final headway. We found that in almost all cases, the final headway is in a very slim interval, the distribution is almost deterministic, with variances between 0.6 and $0.13 \mathrm{sec}^{2}$. Table 2 shows the mean and confidence interval for some values, together with an indication how long the early braking phase in an example run is. (Following times below $70 \mathrm{sec}$ are not interesting, because they lead to braking just after departure.) As discussed by Wendler [3] and verified by simulation, the braking phase determines the final minimum headway time. (The braking phase in this case is more restricting than the accelerating phase). But at low speeds, the communicated train position is a good estimate of its current position - at $80 \mathrm{~km} / \mathrm{h}$, the train tries to report its position once every $111 \mathrm{~m}-$, so that intermittent communication errors do not lead to much delay for the following train.

\section{Conclusion}

In order to estimate the impact of GSM-R communication on the line capacity we introduced a stochastic communication model. We extended this model in order to 
emulate track and train behaviour and conducted simulations of a railway system with a simple track layout by means of two modelling approaches, and compared the results. We found that the results of this newly introduced stochastic model are comparable to those obtained by applying a sophisticated modelling tool. As the tool RUT is being used in practice, we are confident that the models we produced are also close to reality.

The simulation of ETCS level 3 indicates that GSM-R communication failures do not have a severe impact on the capacity of a line. Thus, the deterministic modelling approach of model A (which does not take into account GSM-R communication errors) may be appropriate. These results can even be extended for railway systems equipped with GSM-R communication as it occurs in ETCS level 2 , as long as the braking phase on the track determines the minimum headway time; this holds for traditional, same-size blocks and for the case where blocks are smaller near stations (but not if one also enlarges blocks maximally on open track). We could verify the appropriateness of the deterministic modelling approach with regards to the GSM-R communication by comparing it to a stochastic modelling approach.

We propose, as future work, to evaluate similarly other aspects of railway operation which are represented deterministically for simplicity: are those simplifications also close enough to the (stochastic) real behaviour?

\section{References}

[1] Theeg, G. \& Vincze, B., European train protection systems compared. Signal + Draht, 99(7+8), pp. 35-40, 2007.

[2] Winter, P., ETCS: the european train control system: Requirements and reality. $R T R, \mathbf{5 1}(\mathbf{2 + 3})$, pp. 29-37, 2002.

[3] Wendler, E., Weiterentwicklung der Sperrzeitentreppe für moderne Signalsysteme. Signal + Draht, 87(7+8), pp. 268-273, 1995.

[4] Geiß, G., CIR-ELKE-Pilot Offenburg-Basel. Signal + Draht, 94(1+2), pp. 39-41, 2002.

[5] Jansen, D.N. \& Hermanns, H., Dependability checking with StoCharts: Is train radio reliable enough for trains? QEST 2004: first international conference on the quantitative evaluation of systems, IEEE Computer Society: Los Alamitos, pp. 250-259, 2004.

[6] Sauer, W., RUT: Computer assisted route design. Eisenbahntechnische Rundschau, 48(11), pp. 720-725, 1999.

[7] Euroradio FFFIS : class 1 requirements. http://www.aeif.org/db/docs /ccm/SUBSET-052_v200.PDF 2000.

[8] Performance requirements for interoperability. http://www.aeif.org/db/docs /ccm/SUBSET-041_v200.PDF 2000.

[9] Hermanns, H., Jansen, D.N. \& Usenko, Y.S., From StoCharts to MoDeST: a comparative reliability analysis of train radio communications. Proceedings of the 5th international workshop on Software and performance, ACM Press: New York, pp. 13-23, 2005. 
[10] Brünger, O. \& Dahlhaus, E., Running time estimation. Railway Timetable \& Traffic, eds. I.A. Hansen \& J. Pachl, Eurailpress: Hamburg, chapter 4, pp. 5882, 2008.

[11] Jansen, D.N., Hermanns, H. \& Katoen, J.P., A QoS-oriented extension of UML Statecharts. «UML» 2003: the unified modeling language, eds. P. Stevens, J. Whittle \& G. Booch, Springer: Berlin, volume 2863 of Lecture notes in computer science, pp. 76-91, 2003.

[12] D’Argenio, P.R., Hermanns, H., Katoen, J.P. \& Klaren, R., MoDeST: a modelling and description language for stochastic timed systems. Process Algebra and Probabilistic Methods. Performance Modelling and Verification: PAPM-PROBMIV, eds. L. de Alfaro \& S. Gilmore, Springer: Berlin, volume 2165 of $L N C S$, pp. 87-104, 2001.

[13] Deavours, D.D., Clark, G., Courtney, T., Daly, D., Derisavi, S., Doyle, J.M., Sanders, W.H. \& Webster, P.G., The Möbius framework and its implementation. IEEE Transactions on Software Engineering, 28(10), pp. 956-969, 2002. 